\title{
Preparative HPLC separation of bambuterol enantiomers and stereoselective inhibition of human cholinesterases
}

\section{Ivana Gazić • Anita Bosak • Goran Šinko • Vladimir Vinković • Zrinka Kovarik}

Published online: 21 October 2006

(C) Springer-Verlag 2006

Unfortunately, there were some typographical errors in this article. The corrections are given below.

Page 1514, Fig. 1.

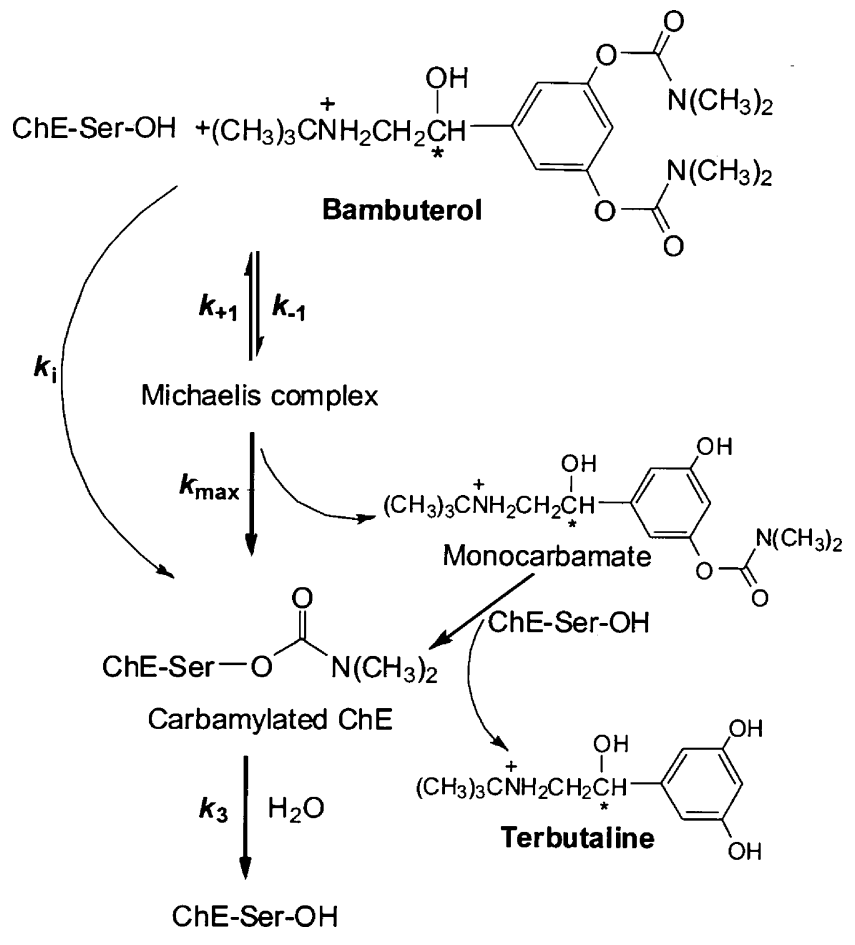

The online version of the original article can be found at http://dx.doi. org/10.1007/s00216-006-0566-3.

I. Gazić • V. Vinković

Ruđer Bošković Institute,

Bijenička cesta 54,

10002 Zagreb, Croatia

\footnotetext{
A. Bosak $\cdot$ G. Šinko $\cdot$ Z. Kovarik $(\square)$

Institute for Medical Research and Occupational Health,

Ksaverska cesta 2,

10000 Zagreb, Croatia

e-mail: zkovarik@imi.hr
}

Page 1515

First paragraph, last line: Reference [8] should read [9]

Right column, line 4: $\mathrm{K}_{\mathrm{i}}$ should read: $K_{\mathrm{i}}$

Equation (3) should read $k_{\mathrm{obs}}=\frac{k_{\max } \cdot i}{K_{\mathrm{i}}+i}$

Equation (4) should read: $k_{\mathrm{i}}=\frac{k_{\max }}{K_{\mathrm{i}}}$

Page 1518, footnote (c) in Table 2 should read: The ratio was $K_{\mathrm{i}(S)} / K_{\mathrm{i}(R)}$

Page 1519 , line $23: \ldots \mathrm{K}_{\mathrm{i}}$ should read: $K_{\mathrm{i}}$ 